\title{
Letter to the Editor
}

\section{Missed retained tick: "Look for the legs"}

\section{Dear Editor,}

Ticks are blood sucking ecto-parasites of vertebrates. They are arachnids from the phylum Arthropoda, subclass Acari, comprising about 800 species. There are two major families, Ixodidae (hard ticks) which have a hard dorsal scutum and Argasidae (soft ticks). ${ }^{1}$ Man is an accidental host. Ticks are vectors of diseases such as rickettsial infections and Lyme disease. India has 106 reported species of ticks. The genera Rhipicephalus, Hyalomma and Haemaphysalis are the commonest with Rhipicephalus microplus, Hyalomma anatolicum and Haemaphysalis spinigera being the commonest species in India. ${ }^{2}$ The major tick borne diseases prevalent in India are Indian Tick Typhus (vector-Rhipicephalus sanguineus), Crimean-Congo haemorrhagic fever (vector-Hyalomma anatolicum) and Kyasanur Forest Disease (vector-Haemaphysalis spinigera). There have also been case reports of Lyme disease from Haryana and an outbreak of the disease in Wayanad, Kerala. We report two cases, where the patients and their primary physicians were unaware of a tick having bitten them and remained attached to the skin.

A 50 year old female patient presented with a painful brown boil on her ear since one week. The patient confirmed to ownership of a pet dog in routine enquiry. The pain started as a mustard sized brown spot and marginally increased in size. She was treated by a general practitioner for a "boil". Dermatological examination revealed a $1 \mathrm{~mm} \times 2 \mathrm{~mm}$, brownish black, convex object, situated on the helix of left ear. Closer examination revealed four pairs of tiny legs under the object, confirming a tick (Fig. 1). With consent, the live tick was carefully extracted with a pair of forceps. Pain was relieved within twenty-four hours. On follow-up at one month, she was asymptomatic, had no eschar and hence no serological test was done. The tick was a hard tick and since the specimen was damaged during extraction, the species could not be identified.

A 48 year old male patient presented with a painful boil on his left hand since four days. There was history of walking through foliage prior to onset of the lesion. The "boil" marginally increased in size over four days. He had no relief with antibiotics and pain killers. Dermatological examination revealed a single, bluish grey, oval, $3 \mathrm{~mm} \times 2 \mathrm{~mm}$ convex object, adherent to the fourth web space of left hand (Fig. 2). Closer examination revealed four pairs of legs confirming a tick. With consent, it was carefully removed with a pair of forceps. The tick measured $3 \mathrm{~mm} \times 2 \mathrm{~mm}$ in size. In both cases, it was an Ixodid tick (hard tick) characterized by the hard chitinous dorsal surface. The
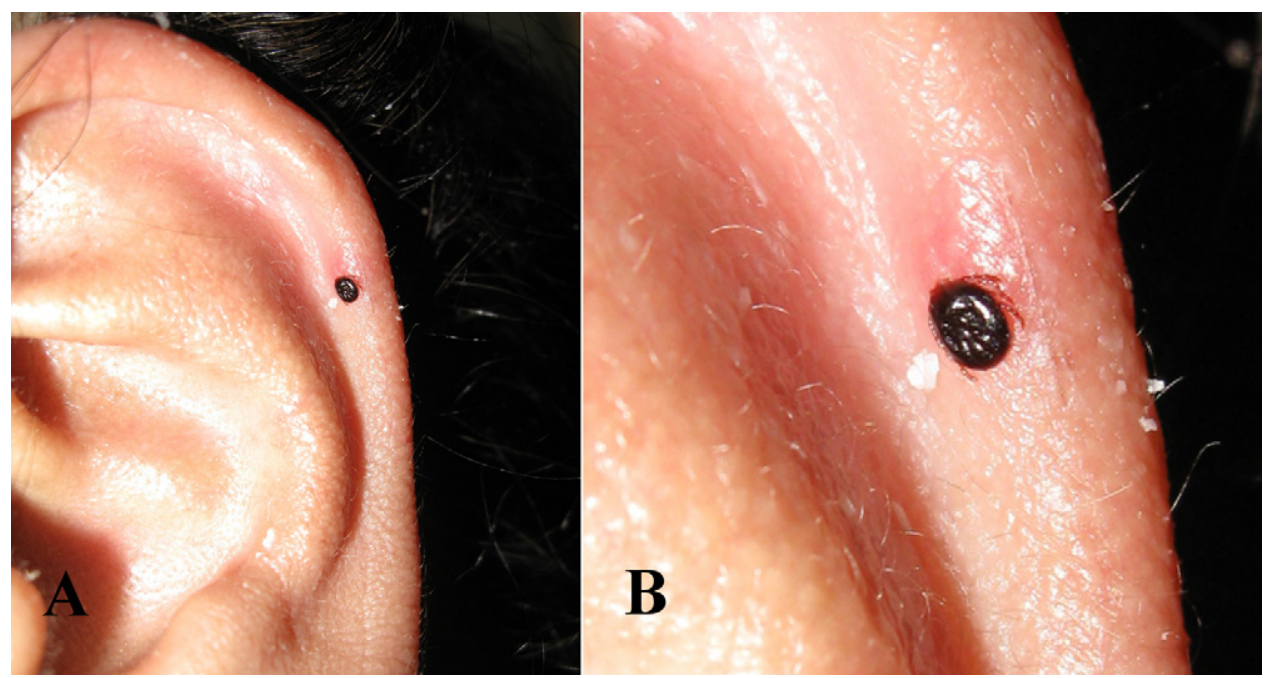

Fig. 1 - (A) Retained tick on the helix of left ear. (B) Close up of tick reveals the legs. 

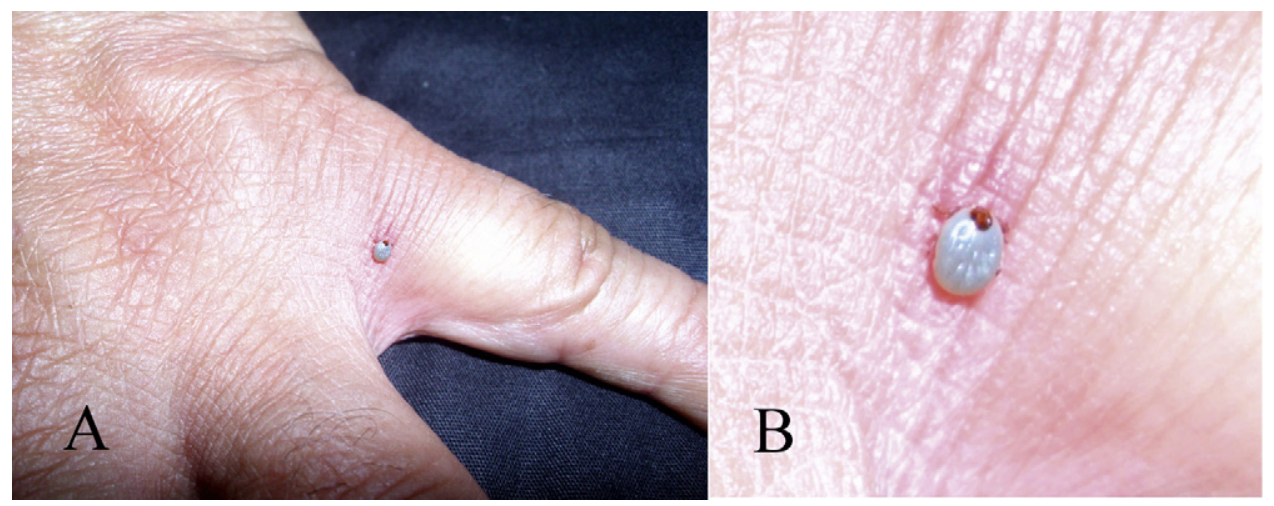

Fig. 2 - (A) A small tick holding on to the fourth web space of left hand. (B) Legs of the tick seen tucked underneath.

patient did not report for follow-up. The tick specimen was identified as Hyalomma aegyptium. Hyalomma is a common genus of hard tick encountered in India. However, Hyalomma anatolicum is the commoner species seen. ${ }^{2}$

Many patients are unaware of ticks biting them. They either notice it as a "boil" or a new mole ${ }^{3}$ which is asymptomatic or associated with pain. The ticks are missed unless they are big enough. The key point is to observe the tiny legs tucked away under the "object". History of contact with animals or brushing through foliage is important. Ixodid ticks attach firmly to the skin up to a week or more and get engorged. They are not dislodged easily. Agasid ticks feed briefly and are not usually encountered.

Ticks have to be removed early, since the risk of transmission of disease increases beyond 24 hours of attachment. The common method is using an angulated forceps with steady traction, with care to keep mouthparts intact. ${ }^{4}$ After removal, the patient needs to be observed for at least 30 days for signs and symptoms of tick borne disease. Prophylaxis with a single oral dose of Doxicycline $200 \mathrm{mg}$ following a tick bite in a hyperendemic region proved effective but controversies exist. ${ }^{5}$

The aim of this letter is to sensitise dermatology residents and other clinicians about the possibility of missing a small retained tick unless one looks for the legs carefully, which are invariably tucked underneath.

\section{R E F E R E N C E S}

1. Schwartz RA, Steen CJ. Arthropod bites and stings. In: Goldsmith LA, Katz SI, Gilchrest BA, Paller AS, Leffell DJ, Wolff $\mathrm{K}$, eds. In: Fitzpatrick's dermatology in general medicine 8th ed. Mc-Graw Hill; 2012:2604-2605.

\section{Letter to the Editor}

\section{Dear Editor,}

As a researcher in the field of high altitude with published body of work and currently posted to High altitude area, I read with considerable interest, the original article titled "Incidence of
2. Ghosh S, Nagar G. Problem of ticks and tick-borne diseases in India with special emphasis on progress in tick control research: a review. J Vector Borne Dis. 2014;51:259-270.

3. Parasitic infestations, stings and bitesJames WD, Berger TG, Elston DM, eds. In: Andrews' diseases of the skin 11th ed. Saunders Elsevier; 2011:441-442.

4. Roupakias S, Mitsakou P, Nimer AA. Tick removal. J Preu Med Hyg. 2011;52:40-44.

5. Nadelman RB, Nowakowski J, Fish D, et al. Prophylaxis with single-dose doxycycline for the prevention of Lyme disease after an Ixodes scapularis tick bite. N Engl J Med. 2001;345:79-84.

Col S. Radhakrishnan* Senior Adviser (Derm \& STD), Command Hospital (Air Force), Bengaluru 560007, India

Air Vice Marshal D.C. Agarwal, vsm (Retd) Ex-Commandant, Command Hospital (Air Force), Bengaluru, India

Manjunath V. Marigeri Postgraduate Trainee (Derm \& STD), Command Hospital (Air Force), Bengaluru, India

*Corresponding author: Tel.: +91 9449275068 E-mail address: drsrkskin@gmail.com (S. Radhakrishnan)

Received 9 May 2015 Available online 17 October 2015

http://dx.doi.org/10.1016/j.mjafi.2015.08.005

0377-1237/

(C) 2015 Published by Elsevier B.V. on behalf of Director General, Armed Forces Medical Services. high altitude pulmonary edema in low-landers during reexposure to high altitude after a sojourn in the plains", by Apte et al. published in Med J Armed Forces India 2015;71:214-220. ${ }^{1}$

Planning and conduct of a prospective cohort study is fraught with various challenges due to inherent limitations of our data management techniques. Moreover, data collection in 